\title{
Free Vibration of a Carbon Nanotube-based Mass Sensor
}

\author{
Payam Soltani ${ }^{1, a}$, Omid Pashaei ${ }^{1, ~ b}$, Mohammad Mehdi Taherian $^{2, c}$ \\ Anoushiravan Farshidianfar ${ }^{3}$ \\ ${ }^{1}$ Department of Mechanical Engineering, Semnan branch, Islamic Azad University, Semnan, Iran \\ ${ }^{2}$ Department of Mechanical Engineering, Semnan branch, Islamic Azad University, Young \\ Researchers Club, Semnan, Iran \\ ${ }^{3}$ Department of Mechanical Engineering, Ferdowsi University of Mashhad, Mashhad, Iran \\ asoltanip2@asme.org, bo.pashaee_narenj@yahoo.com, cm.mehdi.taherian@gmail.com
}

Keywords: Single-walled carbon nanotube (SWCNT), Mass sensor, Nonlocal theory, Euler-Bernoulli beam theory, Shift frequency (SF)

\begin{abstract}
In this paper, nonlocal Euler-Bernoulli beam theory is applied to investigate the dynamical behavior of a single-walled carbon nanotube (SWCNT) with an extra added nanoparticle. The SWCNT is assumed to be embedded on a Winkler-type elastic foundation with cantilever boundary condition. This configuration can be used as a nano-mass sensor which works on the basis of the changing the natural frequencies. The results show that the added mass causes an obvious increase in sensitivity of SWCNT-based nano-mass sensor, especially for stiff mediums, small nonlocal parameters, and stocky SWCNTs.
\end{abstract}

\section{Introduction}

Because of superior mechanical, chemical and thermal properties, carbon nanotubes (CNTs) have many new applications in technological and biomedical devices such as nano-sensors, nano-bio sensors, and nano-mass sensors [1-3]. Recently, Mass detection based on the resonating nanomechanical tools has been subjected of growing interests. The essence of mass sensing in a resonator is based on this fact that the vibration behavior of the resonator is related to changing the total mass of the system and the attached mass causes the resonant frequency to change. The theoretical theories such as elastic continuum mechanics, as well as molecular dynamics (MD)simulations are used for simulating vibration behaviors of CNTs. The MD simulation involves complex computational processes and is still formidable and expensive, especially for large-scale nanostructures. Since, the results of continuum-based modeling show a good agreement with MD simulations, continuum elastic theories are widely utilized to simulate the vibration of CNTs [4]. For example, the potential of SWCNT as a mass sensor is investigated using continuum mechanics theory [5] and the effects of attached buckyballs on the longitudinal vibration of SWCNTs have been presented on the basis of a nonlocal continuum model [6]. Moreover, the resonant frequencies of a cantilevered and a bridged SWCNT were examined using finite element method (FEM) and as a mass sensor, the corresponding shifts of the frequencies is analyzed [7].

This paper makes the effort to study the linear transverse vibration of a SWCNT with an attached nanoparticle. The Euler-Bernoulli beam and nonlocal elasticity theory are applied to analyze the vibrational behavior of the cantilever SWCNT embedded in a Winkler-type medium. The equation of motion is solved using the Galerkin method. The Effects of the added mass, stiffness of the surrounding medium, aspect ratio of the SWCNT, and the nonlocal parameter on the resonant frequency shift are considered and discussed widely.

\section{Modeling}

Fig. 1 Shows a SWCNT with an attached mass $m$ in location $X_{m}$ that is modeled as an EulerBernoulli beam . $E, \rho, A, L$ show the Young's modulus, density, cross-section area, and length of the SWCNT, respectively while the outer diameter and the thickness of the nanotube are $d_{o}$ and $h$, in 
that order. The SWCNTs is fixed at one end and free at the other end that known as a clamped-free (C-F) or cantilever boundary condition. Furthermore, the surrounding elastic medium is simulated as a Winkler-type foundation which represents the stiffness of the medium by Winkler constant $K$.

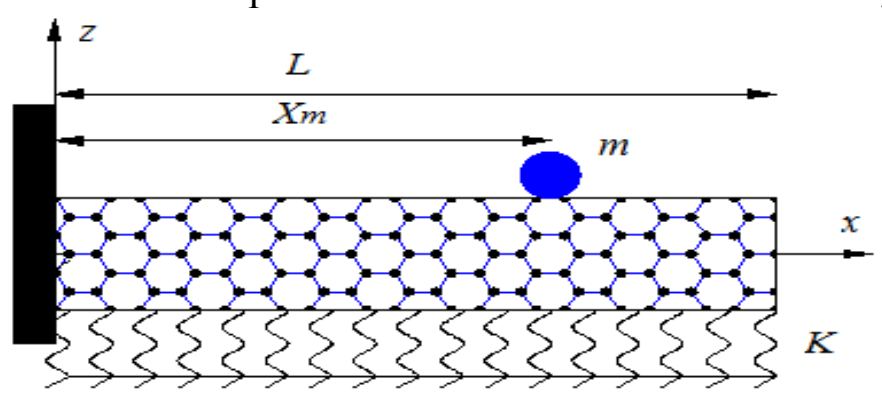

Fig. 1.The cantilever SWCNT embedded in an elastic medium with an attached mass.

By using the Hamilton's principle and based on the nonlocal Euler-Bernoulli beam theory, the governing equation of motion can be written in terms of displacement as follows:

$$
\begin{aligned}
& E I \frac{\partial^{4} w(x, t)}{\partial x^{4}}+\rho A \frac{\partial^{2} w(x, t)}{\partial t^{2}}+K w(x, t)+m \delta\left(x-X_{m}\right) \frac{\partial^{2} w(x, t)}{\partial x^{2}} \\
& -\left(e_{0} a\right)^{2} \frac{\partial^{2}}{\partial x^{2}}\left[\rho A \frac{\partial^{2} w(x, t)}{\partial t^{2}}+K w(x, t)+m \delta\left(x-X_{m}\right) \frac{\partial^{2} w(x, t)}{\partial x^{2}}\right]=0
\end{aligned}
$$

In the above equation, $\delta($.$) is Dirac-delta function, w(x, t)$ is the transverse displacement component in the $x-z$ plan. $x$ and $t$ define axial coordinate and time, respectively. In addition, $e_{0} a$ represents a nonlocal parameter where $e_{0}$ and $a$ are the material constant and an internal characteristic length, in that order. It should be noted that when the nonlocal parameter $e_{0} a$ and the attached mass $m$ are set to be zero in Eq. 1, the local equation of motion of an Euler-Bernoulli beam is easily obtained [7].

To determine the fundamental frequency of the model, the one-approximation Galerkin method is utilized. In modal form, the transverse dynamic displacement of the SWCNT with C-F boundary condition is written as follows [8]:

$$
w(x, t)=W(x) \cdot q(t), \quad W(x)=\cos \left(\frac{1.875 x}{L}\right)-\cosh \left(\frac{1.875 x}{L}\right)-0.734\left[\sin \left(\frac{1.875 x}{L}\right)-\sinh \left(\frac{1.875 x}{L}\right)\right]
$$

Where $q$ and $W$ are unknown time-dependent generalized coordinates and the eigenmodes of an undamped different boundary condition beam, respectively.

Applying Eq. 2 into Eq. 1 and multiplying both sides of the resulting equation with $W$, then integrating it over the interval $[0, L]$ with considering the orthogonally condition and general properties of Dirac-delta function [9], the differential equation of the first mode of the generalized deflection can be calculated as:

$$
\frac{d^{2} q(t)}{d t^{2}}+\frac{K_{t}}{M_{t}} q(t)=0
$$

Where $K_{t}$ and $M_{t}$ are the total equivalent stiffness and total effective mass of the vibrational system, respectively.

\section{Numerical results}

In the present study, the fundamental frequency of a cantilever nano-mass sensor $f_{n}=(1 / 2 \pi) \sqrt{K_{t} / M_{t}}$ is obtained according to the nonlocal Euler-Bernoulli beam model. $K_{t}$ and $M_{t}$ for $X_{m}=L$ are written as:

$$
\begin{aligned}
& K_{t}=\left(\frac{0.6 e_{0} a^{2}}{L}-0.5 L\right) K-\frac{3.4 E I}{L^{3}} \\
& M_{t}=\left(\frac{0.6 e_{0} a^{2}}{L}-0.5 L\right) \rho A+\left(-2.3 \text { Heaviside }(L)-\frac{0.0005 \text { Heaviside }(L) e_{0} a^{2}}{L^{2}}+\frac{0.0002 e_{0} a^{2}}{L^{2}}+1.1\right) m
\end{aligned}
$$


The Heaviside $(x)$ is the step function that is known as the anti-derivative of Dirac $(x)$. According to the function definition, Heaviside $(L)$ is equal to unit because the Length of SWCNT $L$ larger than zero.

The mass of the attached nanoparticle can influence the resonant frequency efficiently and is considered here as a key parameter consequently. The geometrical and mechanical properties of the nanotube are assumed approximately corresponds to a $(16,12)$ SWCNT [10]. Moreover, the Winkler medium constant, nonlocal parameter, and aspect ratio are taken as: $K=1 \mathrm{Mpa}, e_{0} a=2 \mathrm{~nm}$, $L / d_{o}=20$ [11].

The fundamental frequency of a cantilever SWCNT with a tip mass $\left(X_{m} / L=1\right)$ is given as a function of mass ratio $\left(M R=m / m_{r}\right)$ in Fig. 2, where $m_{r}$ is the mass of a carbon nucleus. Since increasing the mass of the attached nanoparticle increases the total mass of the system $M_{t}$, the fundamental frequency decreases as it is shown and this frequency reduction exaggrates for nanoparticles with lagrer $M R$.

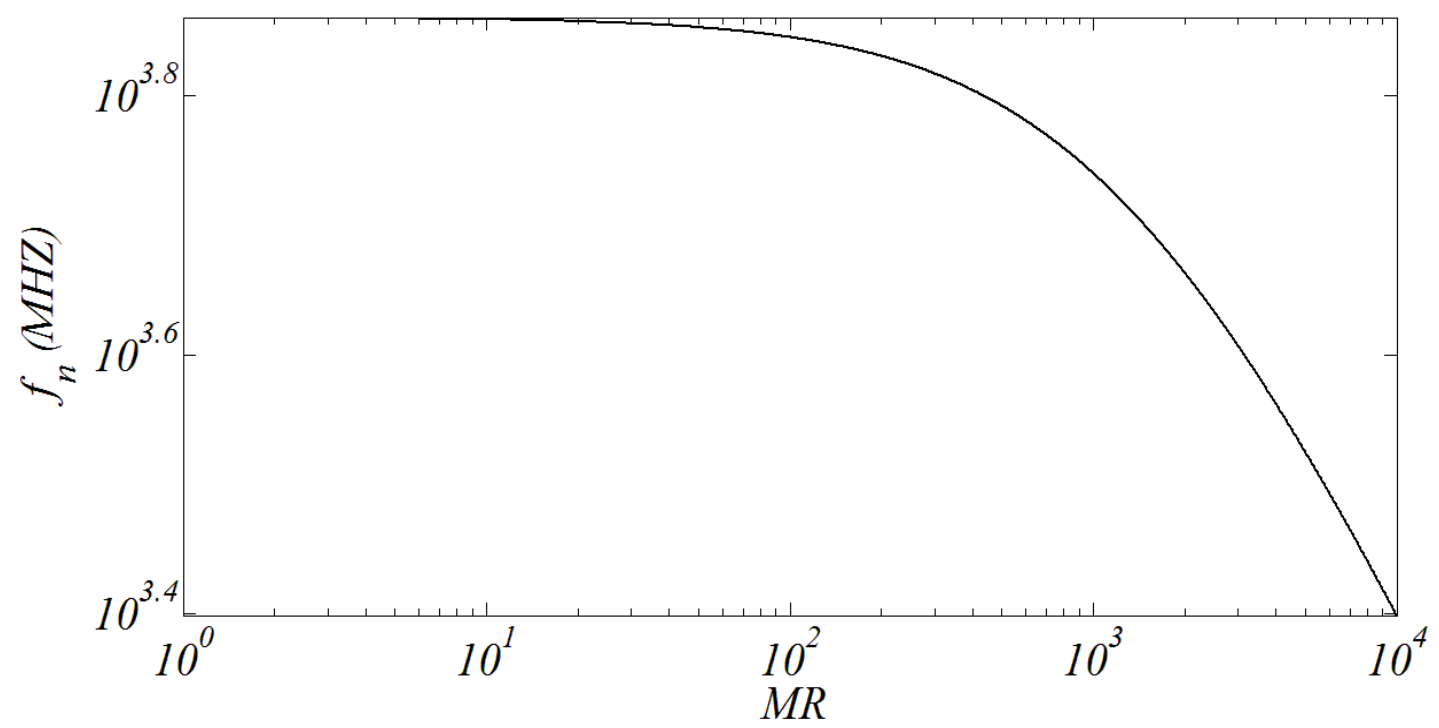

Fig. 2.The fundamental frequency of $\operatorname{SWCNT} f_{n}$ against the mass ratio $M R$

As previously mentioned, the mass sensing with a SWCNT-based mass sensor is based on the fact that the added mass causes a shift to the resonant frequency of the resonator. To gain a better understanding of this fact and to explore the net effect of the attached mass on the resonant frequency of the mass sensor, the shift of frequency $(S F)$ is defined as a parameter that indicates the changes of frequency for the SWCNT with no added mass $(m=0)$ in comparison to the SWCNT containing the attached mass $(m \neq 0)$ as follow:

$$
S F=\left.f_{n}\right|_{(m=0)}-\left.f_{n}\right|_{(m \neq 0)}
$$

Figs. 3-5 show the shift of frequency $S F$ as a function of the mass ratio $M R$, while the impacts of a single specific parameter have been studied in each figure. It can be seen from all these figures that $S F$ increases with increasing the $M R$. Therefore, a high mass sensitivity is revealed for high attached masses. Moreover, It should be noted that for a very large mass (more than $10^{4}$ times greater than the mass of carbon nucleus) do not need a nanotube to be sensed because these masses are larger than the mass of the nanotube.

Fig. 3 shows the influence of the surrounding elastic medium on the $S F$ versus the $M R$. Results demonstrate that with increasing the stiffness medium, due to the Winkler modulus $K$, the shift of frequencies significantly increase. This indicates that as the nanotube vibrates in a stiffer medium, the total stiffness of the system $K_{t}$ is increased and the higher mass sensitivity occurred. 
The $S F$ parameter is highly sensitive to the dimensions of the SWCNT. It is clear from the Fig. 4 that for long and slender SWCNTs with a high aspect ratio $L / d_{o}$, the $S F$ declines and the effects of attached mass on the rise of frequency are reduced.

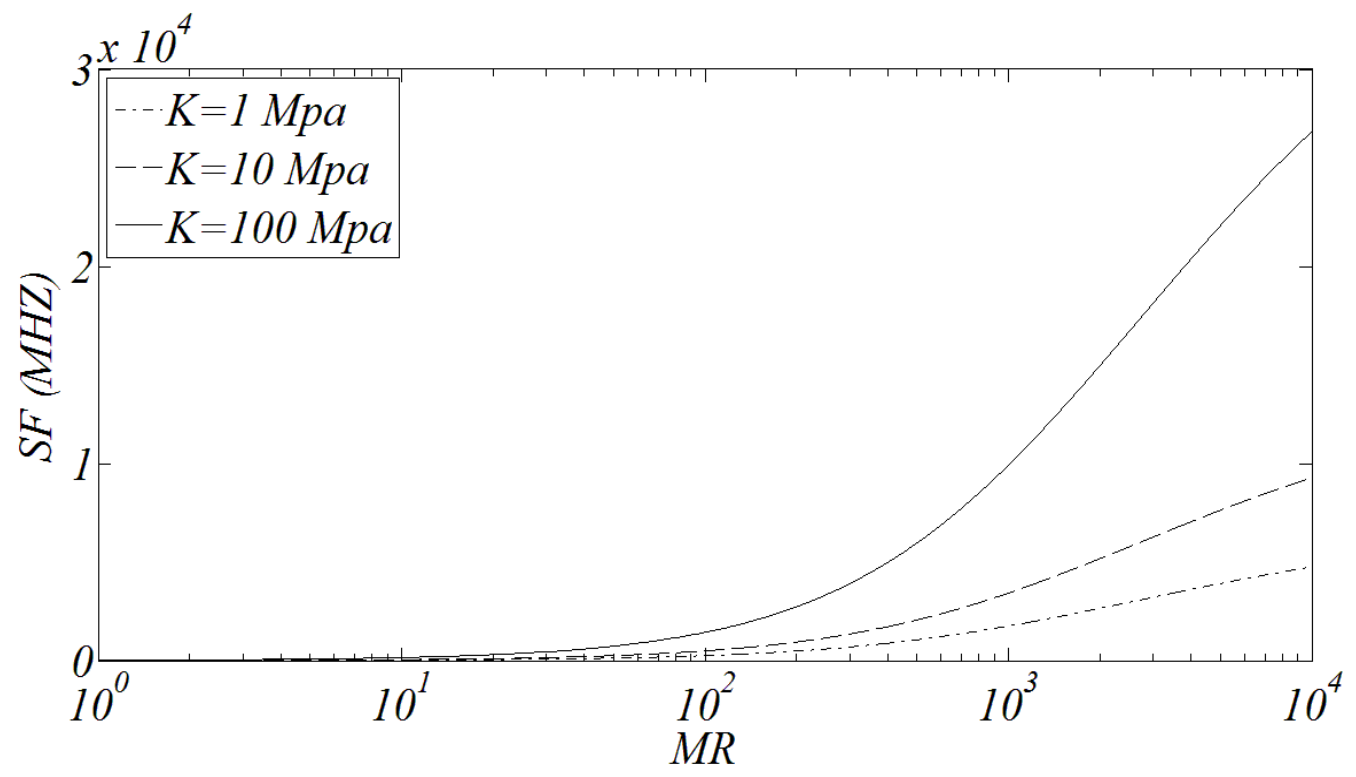

Fig. 3. The shift of frequency $S F$ against the mass ratio $M R$ for different values of the Winkler modulus $K$.

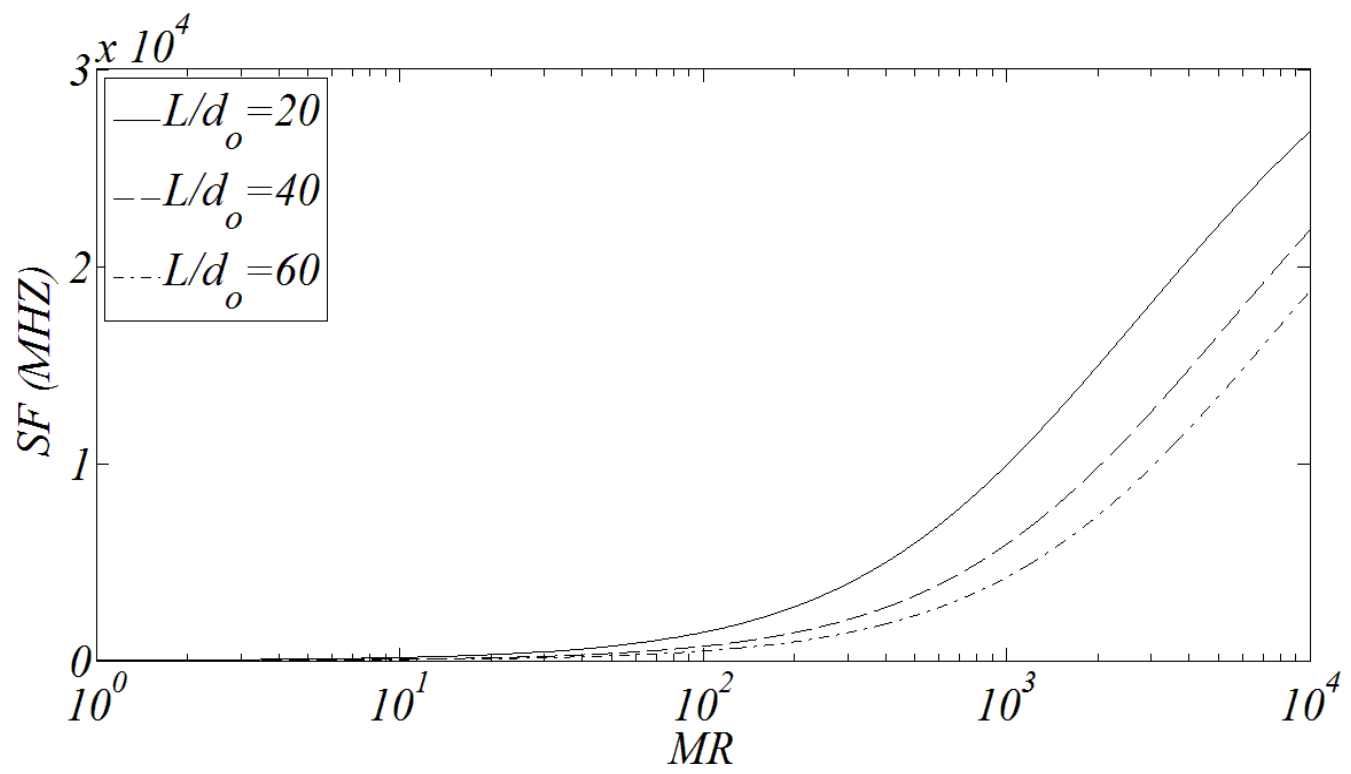

Fig. 4. The shift of frequency $S F$ against the mass ratio $M R$ for different values of the aspect ratio $L / d_{o}$.

As the size of SWCNTs is very small, it is significant to regard the small-scale effect. The local or classical continuum mechanic cannot predict accurately the behavior of nanoscale materials. Unlike the local theory, the nonlocal elasticity observes that the stress at a given point in a body depends not only on the strain at that point but also on those at all points of the body[12]. Fig. 5 illustrates the importance of the nonlocal elasticity and nanoscale effects in the $S F$. The nonlocal elasticity theory causes the SWCNT becomes more flexible and reduce the stiffness of this structure. Therefore, with an increase in SWCNT stiffness by increasing the nonlocal parameter $e_{0} a$, the $S F$ decreases consequently. It can be seen that the larger shift of frequency occurred at the lower values of nonlocal parameter and the nonlocal theory predicts a lower sensitivity for the nano-mass sensor compared to the local ones. 


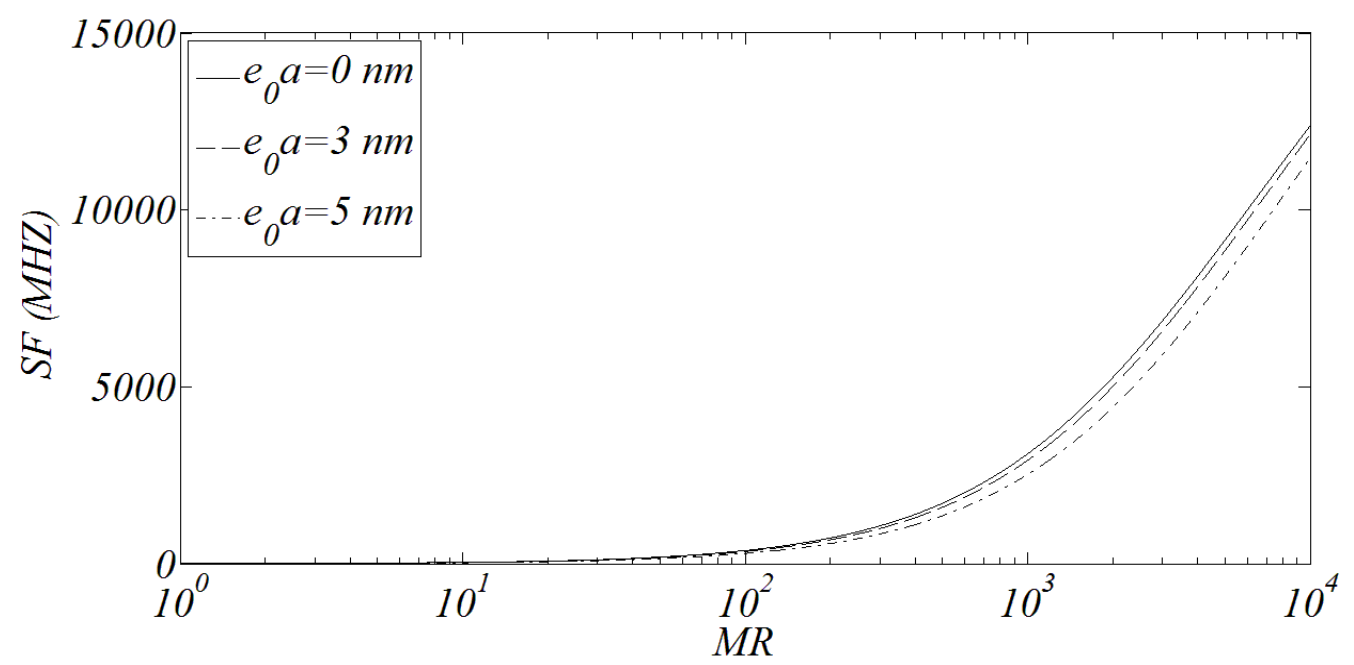

Fig. 5. The shift of frequency $S F$ against the mass ratio $M R$ for different values of the nonlocal parameter $e_{0} a$.

\section{Conclusion}

Based on the nonlocal continuum theory, the dynamical behavior of an embedded cantilever SWCNT carrying a nanoparticle was performed. The obtained governing equation of motion is solved using the Glerkin method, and the natural frequency was calculated. The results indicate that the resonant frequencies increase by an increase in the mass of the nanoparticle. This shift of frequency illustrated the sensitivity of the SWCNT-based mass sensor and was characterized as a parameter. Detailed results demonstrated that the sensor sensitivity rises by increasing the mass ratio, especially for stocky SWCNT, with stiff foundation and the small values of the nonlocal coefficient.

\section{References}

[1] N. D. Hoa, N. Van Quy, Y. Cho, and D. Kim, Sensors and Actuators B: Chemical 135 (2009) 656.

[2] L. G. Carrascosa, M. Moreno, M. A. lvarez, and L. M. Lechuga, Trends in Analytical Chemistry 25 (2006) 196.

[3] J. Arcamone, G. Rius, G. Abadal, J. Teva, N. Barniol, and F. Pe'rez-Murano, Microelectronic Engineering 83 (2006) 1216.

[4] S. K. Georgantzinos and N. K. Anifantis, Physica E: Low-dimensional Systems and Nanostructures 42 (2010) 1795.

[5] R. Chowdhury, S. Adhikari, and J. Mitchell, Physica E: Low-dimensional Systems and Nanostructures 42 (2009) 104.

[6] T. Murmu and S. Adhikari, Mechanics Research Communications 38 (2011) 62.

[7] A. Y. Joshi, S. P. Harsha, and S. C. Sharma, Physica E: Low-dimensional Systems and Nanostructures 42 (2010) 2115.

[8] S. Rao, Vibration of continuous systems, Wiley, Hoboken, New Jersey, 2007.

[9] M. Simsek, Physica E: Low-dimensional Systems and Nanostructures 43 (2010) 182.

[10] S. S. Gupta, F. G. Bosco, and R. C. Batra, Computational Materials Science 47 (2010) 1049.

[11]L. L. Ke, Y. Xiang, J. Yang, and S. Kitipornchai, Computational Materials Science 47 (2009) 409.

[12]A. C. Eringen, Journal of Applied Physics (1976) 


\section{MEMS, NANO and Smart Systems}

10.4028/www.scientific.net/AMR.403-408

Free Vibration of a Carbon Nanotube-Based Mass Sensor

10.4028/www.scientific.net/AMR.403-408.1163 